\title{
Resonance scattering of a transverse sound wave from a planar crystal defect
}

\author{
A.Kosevich ${ }^{1}$, D.Semagin ${ }^{2}$ \\ 1 B.Verkin Institute for Low Temperature Physics and Engineering of the \\ National Academy of Sciences of Ukraine, 47 Lenin Av., UA-310164 \\ Kharkiv, Ukraine \\ 2 Kharkiv State University, 4 Svobody Sq., 310077 Kharkiv, Ukraine
}

Received May 21, 1998

\begin{abstract}
Resonance scattering of a monochromatic transverse acoustic wave of the Rayleigh polarization from an isotop defect layer is considered. The phase velocity $c$ of the incident wave along the defect plane is assumed to lie in the interval $c_{l}>c>c_{t}$. It is shown that the phenomena of the total reflection of the transverse wave from the thin plane defect and the total transition take place for the frequencies depending on the defect parameters. The former can always be observed for a heavy defect and never for a light one. The latter is possible for both light and heavy defects.
\end{abstract}

Key words: scattering, transverse wave, point defect, planar defect

PACS: $71.70 . F k$

When we speak about a planar defect in an elastic medium or crystal lattice, we mean a thin layer of a few atomic distances thick, whose elastic properties differ from those of the host material.There is a number of planar defects in real crystals: grain or twinning boundaries, stacking faults, dislocation walls are typical examples of planar defects.

In the theory of elasticity a planar defect is assumed to be concentrated at some plane and the presence of a planar defect in an elastic medium is associated with boundary conditions at some interfaces for the equation of motion of a non-perturbated medium. In publications $[1,2]$ which were among the first papers devoted to the problem, the boundary conditions consisted in a local perturbation of the elastic moduli at the interface. The result showed the appearence of a local vibration frequency below the lower edge of a transverse vibration band and also features of the transverse spectrum below the edge of a longitudinal band.

A more complete description of the dynamics of an elastic planar defect can be given on the basis of the following procedure. An elastic sandwich $A-B-A$ with the usual boundary conditions at the iterfaces $A-B$ and $B-A$ was considered 
and futher approximation $k h \ll 1$ was used ( $k$ is a two-dimentional wave vector in the defect plane, $h$ is a real thickness of the $B$ layer). Such consideration is used, for exaple, in [3].

Our interest in the scattering from the thin defect layer has been caused basically by the recent publication [3] which asserts that the existence of frequencies corresponding to the resonance reflection coefficient could reach the value equal to unity.

We are interested in solving a specific scattering problem for which the form of the boundary conditions is not significant and also propose to make use of the simplest type of such conditions. We consider a defect which is a monoatomic layer consisting of isotop atoms with mass $\mathrm{M}$ different from the mass of the host atoms $m$.

If the planar defect is located at the plane $z=0$, the dynamic equation for elastic displacement $\boldsymbol{u}(\boldsymbol{x}, t)$ can be written in the form:

$$
\rho \frac{\partial^{2} u_{i}}{\partial t^{2}}-\nabla_{k} \sigma_{i k}=\eta \rho \frac{\partial^{2} u_{i}}{\partial t^{2}} h \delta(z), \quad i=1,2,3 ;
$$

where $\sigma_{i k}$ is a stress tensor, $\eta=\frac{m-M}{m}, h$ is the defect thickness, $\rho$ is the mass density of the medium and $\delta(z)$ is a delta-function.

The r. h. s. of equation (1) is equivalent to the following boundary conditions:

$$
\sigma_{i z}^{+}-\sigma_{i z}^{-}=-\eta \rho h \frac{\partial^{2} u_{i}(0)}{\partial t^{2}}, \quad i=1,2,3
$$

for the dynamic equation of the theory of elasticity in a medium without a defect. Here $\sigma^{+}=\sigma(z=+0), \sigma^{-}=\sigma(z=-0)$.

Consider the problem of the scattering of a monochromatic transverse acoustic wave of Rayleigh polarisation from a thin defect layer in an isotropic medium. If the elastic wave propagates along the $\mathrm{x}$-axis and is polarized in the sagital plane, then the displacement vector $\boldsymbol{u}\left(u_{x}, 0, u_{z}\right)$ is

$$
\boldsymbol{u}=\boldsymbol{u}(z) \exp (i k x-i \omega t)
$$

The vector function $\boldsymbol{u}(z)$ defines dependence on $z$ and consists of two parts (transverse and longitudinal)

$$
\boldsymbol{u}=\boldsymbol{u}^{(t)}+\boldsymbol{u}^{(l)}
$$

It is well known that

$$
\operatorname{rot} \boldsymbol{u}_{l}=\operatorname{div} \boldsymbol{u}_{t} \equiv 0 .
$$

Assume that the phase velocity of the wave $c=\omega / k$ lies between the transverse $c_{t}$ and longitudinal $c_{l}$ sound velocities of the medium $\left(c_{t}<c<c_{l}\right)$.

Under such conditions the transverse wave $\boldsymbol{u}^{(t)}$ is a homogeneous wave in each semispace and a longitudinal wave is localized at the interface. The amplitude of such a wave reaches its maximum at the defect decreasing up to the constant value at infinity. We call these waves pseudosurface waves (PSW). 
If the incident transverse wave falls on the defect from the lower elastic semispace, the displacement field in the both semispaces has the form:

$$
\begin{gathered}
\boldsymbol{u}^{(t)}=\boldsymbol{n}_{0} \exp (i q z)+\boldsymbol{A} \exp (-i q z), \quad z<0, \\
\boldsymbol{u}^{(t)}=\boldsymbol{B} \exp (i q z), \quad z>0, \\
\boldsymbol{u}^{(l)}=\boldsymbol{M} \exp (\varkappa z), \quad z<0, \\
\boldsymbol{u}^{(l)}=\boldsymbol{N} \exp (-\varkappa z), \quad z>0,
\end{gathered}
$$

where $\boldsymbol{n}_{0}$ is a unit polarization vector of the incident transverse wave:

$$
n_{x}^{0}=\cos \theta, n_{z}^{0}=\sin \theta ; n_{z}^{0}=-(k / q) n_{x}^{0} ; c_{t} q=\sqrt{\omega^{2}-k^{2} c_{t}^{2}}, c_{l} \varkappa=\sqrt{k^{2} c_{l}^{2}-\omega^{2}} .
$$

The boundary conditions (2) in the case under consideration are simplified:

$$
\begin{aligned}
& c_{l}^{2}\left(\frac{\partial u_{z}^{+}}{\partial z}-\frac{\partial u_{z}^{-}}{\partial z}\right)=W_{0} u_{z}(0), \\
& c_{t}^{2}\left(\frac{\partial u_{x}^{+}}{\partial z}-\frac{\partial u_{x}^{-}}{\partial z}\right)=W_{0} u_{x}(0),
\end{aligned}
$$

where $W_{0}=\eta h \omega^{2}$. Writing equations (7) and (8) we take into account that the total displacement and its derivative with respect to $x$ are continuous at the plane $z=0$.

The amplitudes $\boldsymbol{A}$ and $\boldsymbol{B}$ of the scattered wave, and $\boldsymbol{M}$ and $\boldsymbol{N}$ of the localized longitudinal vibrations can be calculated on the basis of the boundary conditions (7) and (8) and the conditions of continuity of the total displacement vector. The results of the calculations can be presented in the following form:

$$
\begin{gathered}
A_{x}=\frac{\eta h n_{x}^{0}}{D}\left[2 \varkappa c_{l}^{2}\left(2 k^{2}-\frac{\omega^{2}}{c_{t}^{2}}\right)+\left(k^{2}+\varkappa^{2} \frac{c_{l}^{2}}{c_{t}^{2}}\right) \eta h \omega^{2}\right], \quad A_{z}=(k / q) A_{x} ; \\
B_{x}=-\frac{2 i q n_{x}^{0}}{D}\left[2 \varkappa c_{l}^{2}+\eta h \omega^{2}\right], \quad B_{z}=-(k / q) B_{x} \\
N_{x}=\frac{2 k^{2} \eta h c_{l}^{2} n_{x}^{0}}{D}[\varkappa+i q], \quad N_{z}=(i \varkappa / k) N_{x} ; \\
M_{x}=\frac{2 k^{2} \eta h c_{l}^{2} n_{x}^{0}}{D}\left[i q(1-\varkappa \eta h)-\left(\varkappa+\eta h k^{2}\right)\right], \quad M_{z}=-(i \varkappa / k) M_{x} \\
D=\eta h\left[\frac{\omega^{2}}{c_{t}^{2}}\left(\eta h \omega^{2}+2 \varkappa c_{l}^{2}\right)+\left(\varkappa^{2}-q^{2}\right) \eta h k^{2} c_{l}^{2}\right]-2 i q\left[\left(2 \varkappa c_{l}^{2}+\eta h \omega^{2}\right)-(\eta h k)^{2} c_{l}^{2} \varkappa\right] .
\end{gathered}
$$

As it was expected, the following expression (the conservation law) is valid for all the allowed $c$ :

$$
|\boldsymbol{A}|^{2}+|\boldsymbol{B}|^{2}=1
$$


The condition $\boldsymbol{B}=0$ corresponds to total reflection from the defect and $\boldsymbol{A}=0$ means total transition.

We see that a total reflection exists under the condition

$$
2 c_{l} \sqrt{k^{2} c_{l}^{2}-\omega^{2}}=-\eta h \omega^{2} .
$$

A total reflection is possible provided the defect layer is heavy $(M>m)$.

In the long-wave approximation $(k h \ll 1)$ the phase velocity of the resonance reflected wave $c=\omega / k$ is close to the longitudinal sound velocity $c_{l}$ and defined by the expression

$$
c=c_{l}\left[1-\frac{1}{8}(\eta h k)^{2}\right]
$$

i.e., depends on the perturbation squared: $\Delta c / c \sim(\eta h k)^{2}$.

Bear in mind that condition (15) defines frequency ( or phase velocity $c$ ) of stationary localized longitudinal vibrations satisfying boundary condition (7) in the absence of a transverse component. The total stationary solution, apart from wave (6), includes a standing transverse wave

$$
\begin{gathered}
\boldsymbol{u}^{(t)}=\boldsymbol{a} \sin (q z), \quad z<0, \\
\boldsymbol{u}^{(t)}=0, \quad z>0,
\end{gathered}
$$

which exists only on one side of the plane defect.

The condition of the total transition $(\boldsymbol{A}=0)$ gives

$$
2 c_{l} \sqrt{k^{2} c_{l}^{2}-\omega^{2}}=-\eta h \omega^{2} \frac{k^{2} c_{l}^{2}+k^{2} c_{t}^{2}-\omega^{2}}{2 k^{2} c_{t}^{2}-\omega^{2}} .
$$

We can see that when the defect is heavy $(\eta<0)$, one can obtain the total transition solution $(\boldsymbol{A}=0)$ only under the condition $c_{t}<c<\sqrt{2} c_{t}$. In the longwave approximation $(k h \ll 1)$ the phase velocity $c\left(c \rightarrow \sqrt{2} c_{t}\right)$ is given by the relation

$$
c=\sqrt{2} c_{t}\left[1-|\eta| h k \frac{1-\sigma}{\sqrt{1-2 \sigma}}\right], \quad \sigma=\frac{c_{t}^{2}}{c_{l}^{2}} .
$$

So, the dependence on $(\eta h k)$ is linear.

In the case of a light defect $(\eta>0)$, Eq.(18) leads to a conclusion that the total transition is possible for phase velocities $\sqrt{2} c_{t}<c<c_{l}$ and, when $c \rightarrow c_{l}$ in the long-wave limit $(k h \ll 1)$, we obtain

$$
c=c_{l}\left[1-\frac{1}{8}(\eta h k)^{2} \frac{\sigma^{2}}{(1-2 \sigma)^{2}}\right],
$$

i.e., (20) provides $\Delta c / c \sim(\eta h k)^{2}$, but in a more complex way than in (16).

Therefore, the total reflection can only occur in the case of a heavy defect $(\eta<0)$. The total transition of the transverse wave may occur in the cases of both heavy $(\eta<0)$ and light $(\eta>0)$ defects, but for different frequencies. 


\title{
References
}

1. I.Lifshitz, A.Kosevich. Rep. Progr. Phys., 1966, vol. 29, No 1, p. 217-253.

2. A.Kosevich, V.Khokhlov. Fiz. Tverd. Tela, 1968, vol. 10, No 1, p. 56; 1970, vol. 12, No 9, p. 2570 (in Russian).

3. A.Darinskii, G.Maugin. Wave Motion, 1996, vol. 23, p. 363.

\section{Резонансне розсіяння поперечної звукової хвилі плоским кристалічним дефектом}

\author{
А.Косевич ${ }^{1}$, Д.Семагін ${ }^{2}$ \\ 1 Фізико-технічний інститут низьких температур ім. Б.І.Вєркіна \\ НАН України, 310164 м. Харків, пр. Леніна, 47 \\ 2 Харківський державний університет, \\ 310077 м. Харків, майдан Свободи, 4
}

Отримано 21 травня 1998 р.

\begin{abstract}
Розглянуто резонансне розсіяння на ізотоп-дефектному шарі монохроматичної поперечної звукової хвилі релеївської поляризації. Фазова швидкість $c$ падаючої хвилі вздовж дефектної площини за припущенням лежить в інтервалі $c_{t}<c<c_{l}$. Показано, що явища повного відбиття поперечної хвилі від тонкого плоского дефекту та повного проходження відбуваються при частотах, що залежать від параметрів дефекту. Перше завжди можна спостерігати для важких дефектів та ніколи для легких. Друге явище можливе як для важких, так і для легких дефектів.
\end{abstract}

Ключові слова: розсіяння, поперечна хвиля, плоский дефект

PACS: $70.71 . F k$ 
